Ann. Zootech., 1983, 32 (2), 175-188

\title{
Activité motrice spontanée du chien disposant d'eau et d'aliments ad libitum
}

\author{
Camille OZON, C. DOLISI, J.L. ARDISSON et Dominique CRENESSE \\ avec la collaboration technique de C. BIRot et H. Governatori \\ Laboratoire de Physiologie, Faculté de Médecine de Nice, \\ avenue de Vallombrose, F 06034 Nice Cedex
}

\begin{abstract}
Résumé
L'activité motrice ambulatoire de quatre chiens, disposant d'eau et d'aliments ad libitum, a été enregistrée en continu au moyen d'un détecteur hyperfréquence à effet Doppler.

L'activité moyenne des chiens est importante en début de phase d'éclairement, elle se maintient à un niveau moyen jusqu'à l'extinction de la lumière puis décroît régulièrement pour atteindre son minimum au cours de la dernière heure d'obscurité.

Ce profil présente peu de coïncidences avec ceux de la prise d'eau et d'aliments dont les pics se situent respectivement dans la deuxième partie de la phase d'éclairement et au début de la nuit.

Cependant, chez les quatre chiens, en phase d'éclairement, l'étude des corrélations montre l'existence de fortes liaisons entre le poids des repas et a) la durée de l'intervalle jusqu'au repas suivant, b) la durée totale du repos dans cet intervalle, c) le nombre des alternances repos-activité dans cet intervalle. En phase d'obscurité ces corrélations ne sont pas retrouvées mais, chez deux chiens sur quatre, de nouvelles corrélations s'établissent entre la dimension des prises d'eau et a) la durée du repos dans l'intervalle qui suit, b) la durée de l'activité dans ce même intervalle, c) le nombre des alternances reposactivité.

Ainsi, chez le chien en situation ad libitum, l'organisation des périodes d'activité ambulatoire et de repos est surtout liée au comportement alimentaire en phase d'éclairement et au comportement de prise d'eau en phase d'obscurité.
\end{abstract}

\section{Introduction}

La liaison entre le poids des prises d'aliments et Ia durée des intervalles qui leur font suite a été bien établie chez le rat (Le Magnen \& Tallon, 1966 ; De Castro, 1975 ; Bernstein, 1975), le lapin (SANDerson \& VanderWeele, 1975) et le singe (NATElson \& Bonbright, 1978). Chez le chien (ARdisson et al., 1981), cette liaison a été également retrouvée mais uniquement en phase d'éclairement. La nuit, une liaison analogue est par contre observée avec les prises d'eau.

Chez la plupart des espèces étudiées les rythmes de l'activité et de la prise alimentaire évoluent parallèlement. Ainsi chez le rat (Richter, 1927 ; Hunt \& SCHLos- 
Berg, 1939 ; Finger, 1951 ; Kayser, 1978 ; Armstrong, Coleman \& Singer, 1980) et chez le hamster (Aschoff, Figal.a \& Poppel, 1973) tous les auteurs observent une forte activité nocturne accompagnant des repas volumineux et fréquents. Chez le chien l'activité (O'Connor \& PotTs, 1969 ; Ganz \& KaPpen, 1980) est principalement diurne alors que le rythme de prise alimentaire peut être, selon les sujets, diurne, nocturne ou intermédiaire (ARDisson et al., 1981).

Chez les rongeurs, observés en laboratoire, les évolutions des comportements alimentaire et moteur semblent donc souvent parallèles. Cependant aucune liaison quantitative n'a pu être établie entre leurs rythmes spontanés. Toutefois, BERnsTEIN, en 1975, rapportait chez le rat des résultats faisant état d'une liaison enre la dimension du repas et la durée du repos dans l'intervalle post-prandial.

Devant ces résultats inattendus, il nous a paru intéressant :

1) d'établir le profil de l'activité motrice ambulatoire du chien en semi-liberté et disposant d'eau et d'aliments ad libitum;

2) de rechercher si, comme chez le rat, l'activité évolue parallèlement à la prise alimentaire ou hydrique;

3) d'étudier individuellement chez quatre chiens les relations entre les prises d'eau et d'aliments et les phases d'activité ou de repos qui leur font suite.

\section{Matériel et méthodes}

\section{A. Les animaux}

Quatre chiens adultes mâles ont été utilisés pour cette expérimentation. Le chien $\mathrm{n}^{\prime \prime} 1$ est un bâtard pesant $25 \mathrm{~kg}$, le chien $\mathrm{n}^{\prime \prime} 2$ est également un bâtard pesant $23 \mathrm{~kg}$, le chien $\mathrm{n}^{\circ} 3$ est un animal de race Beagle pesant $15 \mathrm{~kg}$ et le chien $\mathrm{n}^{\circ} 4$ est un bâtard pesant $13,5 \mathrm{~kg}$. Le comportement de ces animaux est observé au laboratoire depuis plusieurs années et ils ont été sélectionnés après un examen vétérinaire et une période d'habituation aux conditions de l'expérience de deux mois minimum.

\section{B. Techniques d'enregistrement}

1. des prises d'eau et d'aliments :

La pièce expérimentale dans laquelle le chien vit pendant la durée de l'expérience ainsi que l'installation permettant l'enregistrement des prises d'eau et d'aliments ont été décrites précédemment (ARDISSON et al., 1981).

2. de l'activité motrice :

Un détecteur hyperfréquence à effet Doppler (Jay électronique type 590), équipé d'un système de protection anti-fluorescence détecte l'activité de l'animal dans toute la pièce. Ce détecteur fonctionne en «tout ou rien» et ses phases d'activation successives sont transcrites en continu sur un enregistreur potentiométrique (Gilson KM). La 
morphologie du tracé obtenu permet de distinguer les mouvements segmentaires du chien, de son activité ambulatoire proprement dite et d'apprécier ainsi les durées d'activité et de repos. Les périodes d'activité retenues pour cette étude correspondent uniquement aux déplacements ambulatoires du chien et aux phases, généralement brèves, d'immobilité en position érigée qu'elles peuvent parfois comporter. Un marqueur d'événement situe avec précision sur le tracé d'activité les débuts et les fins des prises d'eau et d'aliments.

\section{Protocole}

La pièce expérimentale est éclairée artificiellement de $6 \mathrm{~h}$ à $22 \mathrm{~h}$. Les quatre chiens ont subi chacun, pendant une durée totale de 45 jours, le même protocole expérimental. Les durées d'enregistrement continu, durant lesquelles l'animal dispose d'eau et d'aliments (granulés UAR 121) ad libitum, varient de 2 à 5 jours. Elles sont séparées par des journées de sortie, tirées au sort, au cours desquelles le chien se trouve en liberté, de $10 \mathrm{~h}$ à $14 \mathrm{~h}$, sur la terrasse du chenil sans eau ni aliments. A cette occasion le chien est pesé afin de contrôler la stabilité de son poids corporel. Les journées de sortie ainsi que celles émaillées d'un incident technique ne sont pas prises en compte lors de l'exploitation des données. En définitive et pour chaque chien 25 jours utiles sont regroupés pour établir les profils de comportement alimentaire et moteur.

\section{Expression des résultats}

\section{Les profils horaires moyens}

A partir de l'ensemble des données recueillies chez les quatre chiens on a établi : tidiennement ;

- la fréquence horaire moyenne des prises d'eau et d'aliments ;

—. Ia répartition horaire moyenne de l'activité motrice et de sa fréquence.

\section{Les moyennes et erreurs standard à la moyenne (SEM)}

Pour chaque chien, et séparément pour la phase d'éclairement et pour la phase d'obscurité, ont été calculées les moyennes et SEM :

- du poids des prises d'eau et d'aliments;

- de la durée des intervalles séparant deux prises d'eau ou deux prises d'aliments;

- du nombre des phases d'activité et de leur durée totale dans ces intervalles;

- de la durée complémentaire du repos dans ces mêmes intervalles.

\section{Les corrélations linéaires (coefficient « $r »$ )}

Pour chaque chien et séparément pour la phase d'obscurité et pour la phase d'éclairement, les corrélations ont été calculées entre le poids des prises et :

- les interval!es de temps qui leur font suite;

- les intervalles de temps qui les précèdent (les résultats ayant toujours été non significatifs n'ont pas été reportés sur les tableaux):

- la durée de l'activité, le nombre des transitions repos-activité et la durée du repos dans ces mêmes intervalles. 

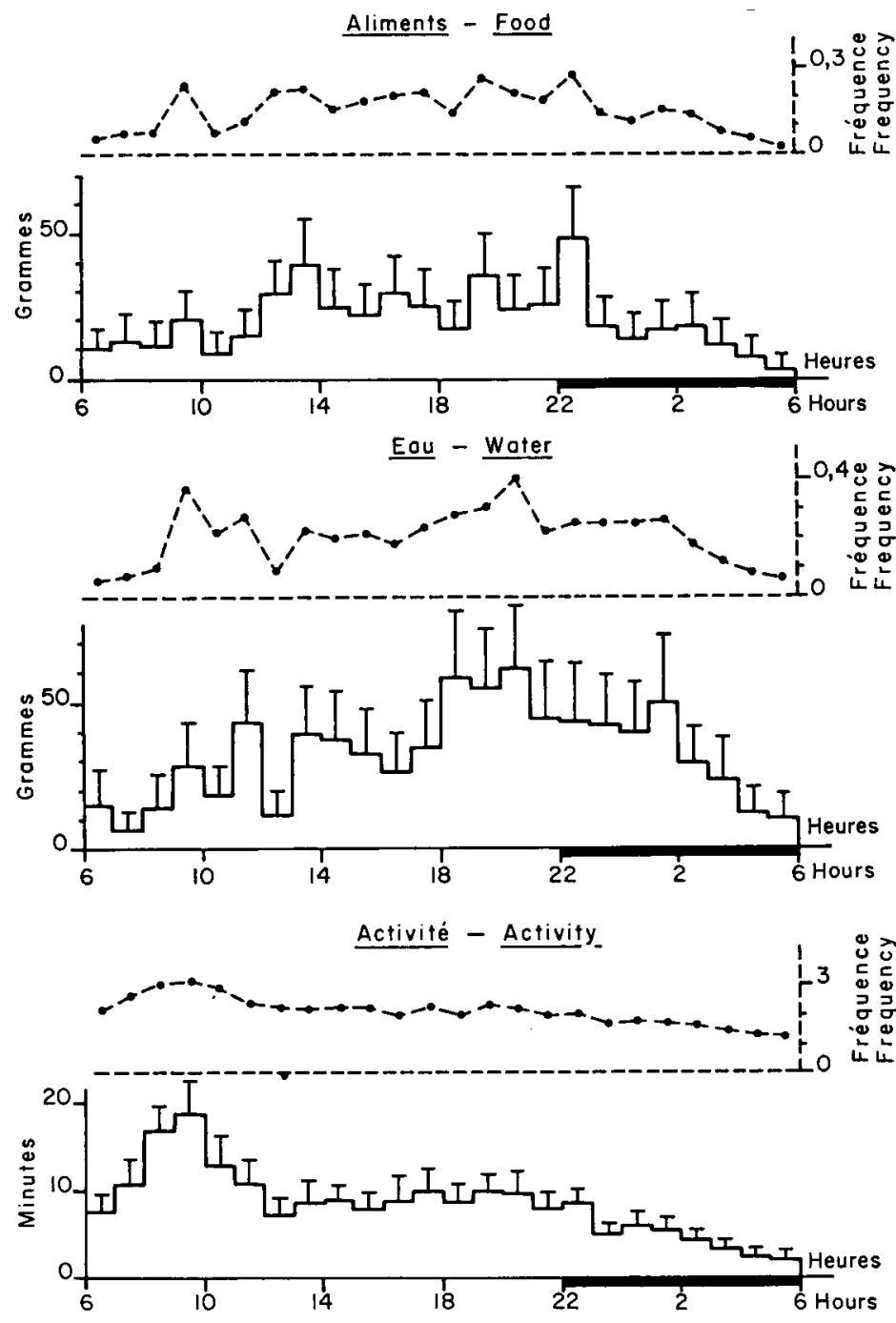

FIG. 1

Profils horaires moyens de la prise d'aliments, de la prise d'eau et de l'activité motrice ambulatoire.

Food intake, water intake and locomotor activity.

- En haut : Histogramme de répartition sur les 24 heures de la quantité moyenne d'aliments ingérée par jour.

Valeurs moyennes de quatre chiens soit 95 jours d'observation.

- Au centre : Histogramme de répartition sur les 24 heures de la quantité moyenne d'eau ingérée par jour.

Valeurs moyennes de quatre chiens soit 95 jours d'observation.

- En bas : Histogramme de répartition sur les 24 heures de la durée moyenne d'activité par jour.

Valeurs moyennes de quatre chiens soit 95 jours d'observation. Les barres verticales indiquent la limite supérieure de l'intervalle de confiance à 95 p. 100. 


\section{Résultats}

\section{A. Les profils horaires moyens}

1. De la prise d'aliments (fig. 1) :

Les prises d'aliments se répartissent inégalement sur l'ensemble du nycthémère. L'ingestion alimentaire, importante durant la deuxième partie de la phase d'éclairement de $12 \mathrm{~h}$ à $21 \mathrm{~h}$, culmine pendant la première heure d'obscurité. Au cours du reste de la nuit elle décroît fortement et se maintient à un niveau réduit pendant la première partie de la phase d'éclairement. La comparaison des deux courbes montre que les prises sont relativement nombreuses mais de faible dimension pendant la tranche horaire 9 à 10 heures.

\section{De la prise d'eau (fig. 1) :}

La courbe des fréquences des prises d'eau présente son maximum dans la tranche horaire 9 à 10 heures. Cependant la période de forte ingestion d'eau se situe entre $18 \mathrm{~h}$ et $2 \mathrm{~h}$, couvrant ainsi les quatre dernières heures de la phase d'éclairement et la première moitié de la nuit. Ensuite, la prise d'eau décroît jusqu'à $8 \mathrm{~h}$, puis tend à remonter tout en présentant un profil d'évolution très irrégulier.

\section{De l'activité motrice (fig. 1) :}

Les courbes de fréquence et de répartition horaire de l'activité motrice présentent des évolutions très concordantes. L'activité motrice, très importante de $7 \mathrm{~h}$ à $12 \mathrm{~h}$ présente un maximum dans la tranche horaire 9 à 10 heures. Elle se maintient à un niveau moyen pendant tout le reste de la phase d'éclairement puis diminue en phase d'obscurité où elle atteint son minimum pendant la dernière heure. Les durées moyennes d'activité s'établissent : à $144 \mathrm{~min} / 24 \mathrm{~h}( \pm 8,2 \mathrm{SEM})$ pour le chien $\mathrm{n}^{\circ} 1$ dont 75,6 p. 100 en phase d'éclairement, à $222 \mathrm{~min} / 24 \mathrm{~h}( \pm 12,1 \mathrm{SEM})$ pour le chien $\mathrm{n}^{\circ} 2$ dont 86,4 p. 100 en phase d'éclairement, à $253 \mathrm{~min} / 24 \mathrm{~h}( \pm 11,1 \mathrm{SEM})$ pour le chien $\mathrm{n}^{\circ} 3$ dont 76,9 p. 100 en phase d'éclairement et à $186 \mathrm{~min} / 24 \mathrm{~h}( \pm 10,0 \mathrm{SEM})$ pour le chien $n^{\circ} 4$ dont 88,0 p. 100 en phase d'éclairement.

Au-dessus de chaque histogramme la courbe en traits interrompus représente l'évolution horaire de la fréquence moyenne des prises (en haut et au centre) et des phases d'activités (en bas).

- Sur l'axe des abscisses le trait épais indique la situation et la durée de la phase d'obscurité.

- Top Histogram (solid line) : $24 \mathrm{~h}$ distribution of the average quantity of food ingested daily.

Curve (dashed line) : $24 \mathrm{~h}$ distribution of eating frequency per day.

- Middle Histogram (solid line) : $24 \mathrm{~h}$ distribution of the average quantity of water ingested daily.

Curve (dashed line) : 24 h distribution of drinking frequency per day.

- Bottom Histogram (solid line) : $24 \mathrm{~h}$ distribution of the average quantity of locomotor activity daily.

Curve (dashed line) : $24 h$ distribution of activity frequency per day.

These curves are an average of four dogs and 95 observation days.

- The vertical bars show the upper limit of the 95 p. 100 confidence interval.

- The dark phase was between 2200 p.m. to 600 a.m. 


\section{Tableau 1}

Prise d'aliments et activité.

Valeurs moyennes et erreurs standards à la moyenne (SEM)

du poids des prises (en grammes), de la durée de l'intervalle après les prises et de la durée d'activité et du repos dans ces intervalles (en minutes).

Coefficient de corrélation " $r$ » entre le poids des prises et les autres paramètres.

\begin{tabular}{|c|c|c|c|}
\hline \multirow{2}{*}{$\frac{\text { Phase }}{{\text { Chien } \mathrm{n}^{\circ}}_{\operatorname{Dog}}}$} & \multicolumn{3}{|c|}{ Eclairement - Lighting } \\
\hline & 1 & 2 & 3 \\
\hline $\begin{array}{l}\text { Quantité moyenne ingérée par } 24 \mathrm{~h} \\
\text { durant chaque phase } \pm \text { SEM } \ldots \ldots \\
\text { Pourcentage de l'ingestion quotidienne } \\
\text { Mean food intake } / 24 \mathrm{~h} \text { during each } \\
\text { phase } \pm \text { SEM. p. } 100 \text { of daily intake }\end{array}$ & $\begin{array}{l}273,75 \pm 20,25 \\
73,4\end{array}$ & $\begin{array}{c}195,96 \pm 16,79 \\
50,2\end{array}$ & $\begin{array}{c}389,76 \pm 13,16 \\
89,8\end{array}$ \\
\hline $\begin{array}{l}\text { Nombre de prises d'aliments } \ldots \ldots \\
\text { Number of food intake }\end{array}$ & 51 & 61 & 91 \\
\hline $\begin{array}{l}\text { Poids moyen d'une prise (P) } \ldots \ldots \\
\mathrm{g} \pm \mathrm{SEM} \\
\text { Average meal weight }\end{array}$ & $121,60 \pm 7,60$ & $79,09 \pm 4,67$ & $117,76 \pm 5,10$ \\
\hline $\begin{array}{l}\text { Durée moyenne de l'intervalle après } \\
\text { une prise (IPost) } \ldots \ldots \ldots \ldots \ldots \ldots \\
\text { min } \pm \text { SEM } \\
\text { Mean length of the post-meal interval }\end{array}$ & $359,50 \pm 38,36$ & $271,33 \pm 14,89$ & $293,78 \pm 19,71$ \\
\hline $\begin{array}{l}\text { Coefficient de corrélation (P-IPost) } \ldots \\
\text { Correlation coefficient }\end{array}$ & $0,411 * *$ & $0,310^{*}$ & $0,420 * * *$ \\
\hline $\begin{array}{l}\text { Durée moyenne d'activité dans l'inter- } \\
\text { valle après une prise (AIPost) } \ldots \ldots \\
\text { min } \pm \text { SEM } \\
\text { Activity mean length in the post-meal } \\
\text { interval }\end{array}$ & $35,22 \pm 3,98$ & $33,13 \pm 3,51$ & $52,51 \pm 3,63$ \\
\hline $\begin{array}{l}\text { Coefficient de corrélation (P-AIPost) } \\
\text { Correlation coefficient }\end{array}$ & 0,177 & $-0,133$ & $0,316^{* *}$ \\
\hline $\begin{array}{l}\text { Nombre moyen des transitions repos- } \\
\text { activité dans l'intervalle après une } \\
\text { prise (NIPost) } \ldots \ldots \ldots \ldots \ldots \ldots \ldots \\
\text { Mean number of rest-activity transi- } \\
\text { tions in the post-meal interval } \\
\end{array}$ & $11,70 \pm 1,15$ & $12,92 \pm 0,79$ & $13,87 \pm 0,98$ \\
\hline $\begin{array}{l}\text { Coefficient de corrélation (P-NIPost) } \\
\text { Correlation coefficient }\end{array}$ & $0,279^{*}$ & $0,283^{*}$ & $0,392 * * *$ \\
\hline $\begin{array}{l}\text { Durée moyenne du repos dans l'inter- } \\
\text { valle après une prise (RIPost) } \ldots \ldots \ldots \\
\text { min } \pm \text { SEM } \\
\text { Rest mean length in the post-meal in- } \\
\text { terval }\end{array}$ & $360,13 \pm 35,33$ & $238,19 \pm 12,71$ & $242,37 \pm 17,44$ \\
\hline $\begin{array}{l}\text { Coefficient de corrélation (P-RIPost) } \\
\text { Correlation coefficient }\end{array}$ & $0,521^{\text {*** }}$ & $0,399 * *$ & $0,414^{* k * *}$ \\
\hline $\begin{array}{rl}* \mathrm{p} & <0,05 \\
* * \mathrm{p} & <0,01 \\
* * * & \mathrm{p}<0,001\end{array}$ & & & \\
\hline
\end{tabular}


Food intake and activity.

Average values ( \pm SEM) of meal weights (grams). post-meal intervals activity and rest during the post-meal interval (min),

for each dog, in light and dark phase.

Correlation coefficient $« r$ between meal weight and the other parameters.

\begin{tabular}{|c|c|c|c|c|}
\hline & \multicolumn{4}{|c|}{ Obscurité - Darkness } \\
\hline 4 & 1 & 2 & 3 & 4 \\
\hline $\begin{array}{c}120,70 \pm 15,26 \\
69,5\end{array}$ & $\begin{array}{c}99,00 \pm 24,00 \\
26,6\end{array}$ & $\begin{array}{c}194,81 \pm 13,57 \\
49,8\end{array}$ & $\begin{array}{c}44,10 \pm 13,16 \\
10,2\end{array}$ & $\begin{array}{c}53,00 \pm 14,46 \\
30,5\end{array}$ \\
\hline 30 & 14 & 53 & 13 & 11 \\
\hline $62,56 \pm 6,16$ & $152,71 \pm 15,41$ & $90,07 \pm 4,59$ & $123,84 \pm 13,08$ & $87,54 \pm 17,95$ \\
\hline $461,13 \pm 52,63$ & $699,85 \pm 68,04$ & $371,46 \pm 32,61$ & $544,30 \pm 42,91$ & $720,73 \pm 81,00$ \\
\hline $0,645^{* * * *}$ & $-0,002$ & $-0,020$ & 0,366 & $-0,178$ \\
\hline $50,81 \pm 8,15$ & $69,23 \pm 12,42$ & $58,90 \pm 9,38$ & $65,67 \pm 10,06$ & $88,56 \pm 10,64$ \\
\hline $0,410^{*}$ & $-0,240$ & $-0,198$ & 0,272 & 0,193 \\
\hline $16,50 \pm 2,09$ & $19,78 \pm 2,32$ & $16,79 \pm 1,84$ & $20,07 \pm 2,33$ & $24,00 \pm 2,91$ \\
\hline $0,632 * * *$ & $-0,012$ & $-0,064$ & 0,214 & $-0,140$ \\
\hline $410,32 \pm 45,60$ & $630,63 \pm 58,52$ & $311,79 \pm 24,66$ & $478,62 \pm 34,83$ & $632,16 \pm 74,03$ \\
\hline $0,671 * * *$ & 0,048 & 0,046 & 0,373 & $-0,222$ \\
\hline
\end{tabular}




\section{TABleaU 2}

Prise d'eau et activité.

Valeurs moyennes et erreurs standards à la moyenne (SEM)

du poids des prises (en grammes), de la durée de l'intervalle après les prises

et de la durée d'activité et du repos dans ces intervalles (en minutes).

Coefficient de corrélation $\| r$ » entre le poids des prises et les autres paramètres.

\begin{tabular}{|c|c|c|c|}
\hline Phase & \multicolumn{3}{|c|}{ Eclairement - Lighting } \\
\hline $\begin{array}{c}\text { Chien } \mathrm{n}^{\circ} \\
\text { Dog }\end{array}$ & 1 & 2 & 3 \\
\hline $\begin{array}{l}\text { Quantité moyenne ingérée par } 24 \mathrm{~h} \\
\text { durant chaque phase } \pm \text { SEM } \ldots . . . \\
\text { Pcurcertage de l'ingestion quotidienne } \\
\text { Mean food intake/24 h during each } \\
\text { phase } \pm S E M . p .100 \text { of daily intake }\end{array}$ & $\begin{array}{c}630,50 \pm 61,50 \\
67,9\end{array}$ & $\begin{array}{c}467,82 \pm 48,99 \\
54,3\end{array}$ & $\begin{array}{c}581,28 \pm 40,74 \\
80,9\end{array}$ \\
\hline $\begin{array}{l}\text { Nombre de prises d'eau } \ldots \ldots \ldots \ldots \\
\text { Number of water intake }\end{array}$ & 68 & 142 & 66 \\
\hline $\begin{array}{l}\text { Poids moyen d'une prise (P) } \ldots \ldots \ldots \\
\mathrm{g} \pm \mathrm{SEM} \\
\text { Average meal weight }\end{array}$ & $219,35 \pm 15,16$ & $82,37 \pm 3,44$ & $221,59 \pm 9,65$ \\
\hline $\begin{array}{l}\text { Durée moyenne de l'intervalle après } \\
\text { une prise (IPost) } \ldots \ldots \ldots \ldots \ldots \ldots \ldots \\
\text { min } \pm \text { SEM } \\
\text { Mean length of the post-meal interval }\end{array}$ & $241,94 \pm 25,45$ & $143,92 \pm 11,28$ & $331,28 \pm 30$ \\
\hline $\begin{array}{l}\text { Coefficient de corrélation (P-IPost) } \ldots \\
\text { Correlation coefficient } \\
\end{array}$ & $0,402 * * *$ & 0,074 & 0,047 \\
\hline $\begin{array}{l}\text { Durée moyerne d'activité dans l'inter- } \\
\text { valle après une prise (AIPost) } \ldots . . . \\
\text { min } \pm \text { SEM } \\
\text { Activity mean length in the post-meal } \\
\text { interval }\end{array}$ & $24,06 \pm 2,94$ & $22,53 \pm 1,96$ & $57,00 \pm 5,06$ \\
\hline $\begin{array}{l}\text { Coefficient de corrélation (P-AIPost) } \\
\text { Correlation coefficient }\end{array}$ & 0,213 & $-0,055$ & 0,037 \\
\hline $\begin{array}{l}\text { Nombre moyen des transitions repos- } \\
\text { activité cars l'intervalle après une prise } \\
\text { (NIFost) .......................... } \\
\text { Mean number of rest-activity transi- } \\
\text { tions in the post-meal interval }\end{array}$ & $7,66 \pm 0,75$ & $7,31 \pm 0,54$ & $14,45 \pm 1,26$ \\
\hline $\begin{array}{ll}\text { Coefficient de corrélation (P-NIPost) } \\
\text { Correlation coefficient }\end{array}$ & $0,321 * *$ & 0,020 & 0,024 \\
\hline $\begin{array}{l}\text { Durée moyenne du repos dans l'inter- } \\
\text { valle après une prise (RIPost) ...... } \\
\text { min } \pm \text { SEM } \\
\text { Rest mean length in the post-meal in- } \\
\text { terval }\end{array}$ & $2: 18,00 \pm 22,96$ & $121,38 \pm 10,06$ & $274,28 \pm 26,06$ \\
\hline $\begin{array}{l}\text { Coefficient de corrélation (P-RIPost) } \\
\text { Correlation coefficient }\end{array}$ & $0,418^{* * *}$ & 0,094 & 0,047 \\
\hline
\end{tabular}


Water intake and activity.

Average values ( $\pm S E M$ ) of drink weights (grams), post-drinking intervals, activity and rest during the post-drinking interval (min),

for each dog, in light and dark phase.

Correlation coefficient $« \boldsymbol{r} \gg$ between drink weight and the other parameters.

\begin{tabular}{|c|c|c|c|c|}
\hline & \multicolumn{4}{|c|}{ Obscurité - Darkness } \\
\hline 4 & 1 & 2 & 3 & 4 \\
\hline $\begin{array}{c}288,85 \pm 39,92 \\
75,0\end{array}$ & $\begin{array}{c}298,00 \pm 57,50 \\
32,1\end{array}$ & $\begin{array}{c}393,99 \pm 26,45 \\
45,7\end{array}$ & $\begin{array}{c}137,48 \pm 29,26 \\
19,1\end{array}$ & $\begin{array}{c}96,30 \pm 22,34 \\
25,0\end{array}$ \\
\hline 36 & 22 & 80 & 17 & 12 \\
\hline $129,63 \pm 9,68$ & $311,95 \pm 16,71$ & $120,14 \pm 4,68$ & $228,17 \pm 10,33$ & $119,25 \pm 17,05$ \\
\hline $351,22 \pm 48,70$ & $633,68 \pm 80,75$ & $192,82 \pm 21,22$ & $515,43 \pm 70,27$ & $634,42 \pm 116,26$ \\
\hline 0,233 & $0, \overline{474^{*}}$ & $0,423 * * *$ & $-0,122$ & $-0,094$ \\
\hline $32,60 \pm 5,58$ & $54,80 \pm 9,98$ & $24,04 \pm 4,45$ & $86,40 \pm 15,69$ & $91,32 \pm 18,54$ \\
\hline 0,135 & $0,464^{*}$ & $0,325 * *$ & 0,162 & 0,119 \\
\hline $11,69 \pm 1,55$ & $16,68 \pm 1,73$ & $7,91 \pm 1,05$ & $28,23 \pm 3,93$ & $24,92 \pm 4,71$ \\
\hline 0,242 & $0,537 * *$ & 0,369 *** & 0,052 & 0,007 \\
\hline $318,62 \pm 43,86$ & $578,87 \pm 72,55$ & $167,58 \pm 17,48$ & $443,86 \pm 64,14$ & $543,09 \pm 100,93$ \\
\hline 0,241 & $0,464 *$ & $0,431 * * *$ & $-0,204$ & $-0,130$ \\
\hline
\end{tabular}




\section{B. Les relations entre la prise d'aliment et l'activité}

Le tableau 1 met en évidence de fortes différences interindividuelles entre les quatre chiens, pour le poids moyen des prises d'aliments et leur nombre, au cours des 25 jours d'observation. Cependant, chez les quatre chiens, les prises sont toujours plus nombreuses et de plus faible dimension en phase d'éclairement qu'en phase d'obscurité. Les intervalles de temps qui suivent les prises d'aliments évoluent comme le poids de ces dernières et il existe une corrélation positive entre la taille des repas et les intervalles de temps qui leur font suite en phase d'éclairement chez les quatre chiens. Dans cette même phase d'éclairement on observe également des liaisons positives entre le poids des repas et le nombre des transitions repos-activité dans les intervalles qui leur font suite et la durée du repos dans ces intervalles.

La durée de l'activité motrice n'est liée au poids des repas que chez les chiens $\mathrm{n}^{\circ} 3$ et $\mathrm{n}^{\circ}$ 4. En phase d'obscurité aucune corrélation significative ne lie la dimension des repas aux valeurs de l'activité ou du repos.

\section{Les relations entre la prise d'eau et l'activité}

Le tableau 2 montre que les dimensions et les fréquences des prises d'eau varient beaucoup d'un chien à l'autre. Cependant, comme pour la prise d'aliment, les prises sont en moyenne plus petites mais p'us nombreuses le jour que la nuit. Les intervalles de temps qui succèdent aux prises d'eau sont corrélés positivement au poids de ces prises en phase d'éclairement chez le chien $n^{\circ} 1$ et en phase d'obscurité chez les chiens $\mathrm{n}^{\prime \prime} 1$ et $\mathrm{n}^{\prime \prime} 2$. Chez ces deux sujets une analyse plus fine montre que des corrélations positives existent avec les trois paramètres qui caractérisent ces intervalles. Ainsi le poids des prises d'eau est-il lié à la durée de l'activité, à celle du repos, et au nombre des transitions repos-activité qui comportent ces intervalles.

\section{Discussion}

Dans les conditions expérimentales décrites, l'activité proprement dite représente, selon les sujets, de 6 à 20 p. 100 de la phase d'éclairement et de 6 à 12 p. 100 de la phase dobscurité. Ce niveau d'activité spontanée peut paraître faible surtout si on le compare aux taux d'activité nocturne rapportés chez le rat par Hunt \& Schlosberg (1939), Mathews \& Finger (1966) et Mony et al. (1978). Il se rapproche cependant des résultats obtenus chez le chien par Hite et al. (1977) et par GANZ \& KaPpen (1980) si l'on ne retient que l'activité proprement ambulatoire. L'évolution de cette activité en phase d'éclairement ne concorde pas avec celle de la prise d'eau et d'aliments. On observe même que l'activité ambulatoire maximale survient en début de phase d'éclairement lorsque la prise d'aliments est minimale. Au cours de la phase d'obscurité une tendance commune ne s'ébauche que pendant la deuxième moitié de la nuit $(2 \mathrm{~h}$ à $6 \mathrm{~h})$ où les trois paramètres présentent leurs niveaux les plus bas. Le chien, animal à activité préférentiellement diurne, s'oppose ainsi aux espèces nocturnes comme le rat (RICHTER, 1927 ; Hunt \& Schlosberg, 1939 ; Mathews \& Finger, 1966) et se rapproche sur ce point des primates (NATElson \& Bonbright, 1978). 
On retrouve, chez les quatre chiens, la relation mise en évidence par LE MAGNEN \& TAlLoN en 1966 entre le poids des repas et lintervalle de temps jusquau repas suivant. Cette liaison n'existe, dans notre étude, que pendant la phase d'éclairement où se produit la plus grande partie de la prise alimentaire. De plus, dans ces intervalles, c'est uniquement avec la durée du repos qu'une liaison plus spécifique et plus intense est retrouvée chez les quatre sujets. En effet, la durée de l'activité post-prandiale est indépendante de la dimension des repas. Par contre, le nombre des transitions reposactivité, c'est-à-dire le nombre des phases de repos est lié, quoique de façon moins intense, à la dimension du repas. Ainsi, en période d'éclairement, la durée totale du repos séparant deux repas dépend du poids du premier repas. L'allongement de cette durée se réalise surtout par une augmentation du nombre des périodes repos et, à un moindre niveau par un accroissement de la durée de ces périodes. En effet, chez les chiens $\mathrm{n}^{\circ} 2$ et 3 il existe une corrélation positive entre le poids des repas et la durée moyenne des périodes de repos dans l'intervalle post-prandial (chien $n^{\circ} 2: r=0,265$, $\mathrm{n}=61, \mathrm{p}<0,05 ;$ chien $\left.\mathrm{n}^{\prime \prime} 3: \mathrm{r}=0,274, \mathrm{n}=91, \mathrm{p}<0,01\right)$. Ces résultats rejoignent les observations complémentaires de FINGER (1951) qui montra linhibition postprandiale de l'activité, de BERNSTEIN (1975), qui établit la relation entre la dimension du repas et la durée du repos post-prandial, et de Danguir, Nicolaidis \& GerarD (1979) qui démontrèrent que ces liaisons sétablissent en obscurité avec les deux intervalles suivant le repas et les durées de sommeil lent, de sommeil rapide et d'évei' qui les constituent. Ainsi, dans la phase de forte charge en nutriments, nocturne chez le rat et diurne chez le chien, la durée du repos ou plus électivement du sommeil chez le rat, est liée à la dimension des repas.

En période d'obscurité ces liaisons ne sont pas retrouvées chez nos quatre chiens. Ceci distingue cette espèce du rat, du lapin et du singe, chez qui la liaison poids-durée de l'intervalle post-prandial a été décrite de nuit comme de jour (LE MAGNEN \& TALLON, 1966 ; Sanderson \& Vanderweele, 1975 ; Natelson \& Bonbright, 1978). Cette divergence pourrait s'expliquer, au moins partiellement, par des différences dans les techniques de mesure et de traitement des paramètres en cause. En effet, en utilisant une méthode d'analyse plus fine, Danguir, Nicolaidis \& Gerard (1979) ont montré, chez le rat, que cette liaison n'est pas significative au début de la phase d'éclairement mais seulement au cours des huit heures couvrant la fin de cette phase et le début de la phase d'obscurité et pendant la nuit.

Nos résultats, indiquent, par contre, que chez le chien en phase d'obscurité ces relations se font avec la prise d'eau chez deux sujets sur quatre. La durée de l'intervalle qui suit une prise d'eau est liée à l'importance de cette prise. L'analyse des paramètres caractérisant cet intervalle montre que la liaison est positive et forte avec la durée du repos dans l'intervalle et avec le nombre des transitions repos-activité. Il existe également chez les deux chiens, une liaison moins intense entre la dimension de la prise d'eau et la durée de l'activité dans l'intervalle. Cette dernière liaison se rapproche de celle rapportée par O'CONNOR \& POTTS (1969) entre l'activité et la consommation d'eau chez des chiens observés dans des cages à métabolisme.

En obscurité, l'augmentation de ces intervalles se fait chez les deux chiens par une augmentation du nombre des périodes de repos et d'activité et de plus, chez le chien $n^{\circ} 2$, par un allongement de la durée moyenne des périodes de repos $(r=0,278$, $\mathrm{n}=80, \mathrm{p}<0,05)$.

Ces résultats suggèrent que, chez le chien en situation ad libitum, l'ajustement des entrées caloriques se ferait essentiellement en phase d'éclairement comme chez les pri- 
mates diurnes (Natelson \& Bonbright, 1978). Au cours de cette phase les repas sont fréquents et leur volume est fortement lié à la quantité d'eau ingérée jusqu'au repas suivant (ARDISSON et al., 1981) ainsi qu'à la durée et au nombre des périodes de repos dans ces mêmes intervalles.

La nuit, les prises d'eau ne sont plus corrélées aux prises d'aliments (ARDisson et al., 1981) mais évoluent selon un profil autonome. Ceci suggère la mise en jeu, au cours de cette phase, de facteurs homéostatiques de la régulation hydrominérale aboutissant à l'ajustement du bilan hydrique. Cette prévalence relative du comportement de prise d'eau rendrait compte de ses liaisons avec la durée et l'organisation des intervalles de temps jusqu'à la prise suivante qui, jusqu'à présent, n'a été décrit que chez cette espèce. Il faut remarquer cependant que ces liaisons sont moins étroites et moins cohérentes que pour la prise d'aliments.

Enfin, deux chiens sur quatre ne présentent aucune liaison significative en phase d'obscurité. Les particularités individuelles de chaque chien, comparables à celles observées chez d'autres espèces (Bernstein, 1975; Levitsky, 1974; Natelson \& BONBRIGHT, 1978), libres de s'exprimer dans la situation ad libitum pourraient être à l'origine d'un phénomène de masquage chez ces sujets. L'étude en situation de contrainte alimentaire ou de restriction hydrique de l'évolution des diverses liaisons observées en situation ad libitum, devrait permettre de mieux apprécier leur importance réelle et leur signification physiologique.

En conclusion, le chien disposant d'eau et d'aliments ad libitum a une activité spontanée à prédominance diurne. La nuit, cette activité persiste tout en décroissant régulièrement pour atteindre son minimum au cours de la dernière heure d'obscurité. L'analyse individuelle des relations entre le poids des prises et les phases d'activité ou de repos qui leur font suite, fait ressortir l'existence de fortes liaisons entre la prise d'aliments et la durée du repos post-prandial en phase d'éclairement et des liaisons moins intenses entre la prise d'eau et l'activité et le repos en phase d'obscurité.

Accepté pour publication en janvier 1983.

\section{Summary}

Spontaneous locomotor activity in dogs with free access to food and water

Locomotor activity of four dogs, with free access to food and water, was recorded continuously with a microwave radar Doppler System.

At the beginning of the light phase, average locomotor activity was of importance and maintained at a mean level until the light was switched off. A regular decrease was then observed with a minimum in the last hour of the dark phase (fig. 1).

This profile shows poor coincidence with drinking and eating patterns whose to levels occurred in the second part of the light phase and at the beginning of the dark phase, respectively (fig. 1).

However, in the light phase, the weight of each meal was significantly correlated with a) the length of the post-meal interval, b) the amount of rest in the post-meal interval, c) the number of alternations in rest-activity in the same interval (tabl. 1).

In the dark phase, these correlations were not found. But, in two dogs the weight of each drink and a) the amount of rest in the post-drink interval, b) the amount of 
locomotor activity in the same interval, c) the number of alternations in the rest-activity, appeared to be positively correlated (tabl. 2).

Therefore, in the dog in an ad libitum situation, spontaneous organisation of activity and resting patterns is essentially linked to eating behavior in the light phase and to drinking behavior in the dark phase.

\section{Références bibliographiques}

Ardisson J.L., Dolisi C., Ozon C., Crenesse D., 1981. Caractéristiques des prises d'eau et d'aliments spontanées chez des chiens en situation ad lib. Physiol. Behav., 26, 361-370.

Armstrong S., Coleman G., Singer G., 1980. Food and water deprivation : changes in rat feeding, drinking, activity and body weight. Neurosci. Biobehav. Rev., 4, 377-402.

Aschoff J., Figala J., Poppel E., 1973. Circadian rhythms of locomotor activity in the golden hamster (Mesocricetus auratus) measured with two different techniques. $J$. comp. Physiol. Psychol., 85, 20-28.

BerNStein I.L., 1974. Post-prandial EEG synchronization in normal and hypothalamically lesioned rats. Physiol. Behav., 12, 535-545.

BERNSTEIN I.L., 1975. Relationship between activity, rest, and free feeding in rats. $J$. comp. Physiol. Psychol., 89, 253-257.

Danguir J., Nicolaidis S., Gerard H., 1979. Relations between feeding and sleep patterns in the rat. J. comp. Physiol. Psychol., 93, 820-830.

De Castro J.M., 1975. Meal pattern correlations : Facts and Artifacts. Physiol. Behav., 15, 13-15.

FIngER F.W., 1951. The effect of food deprivation and subsequent satiation upon general activity in the rat. J. comp. Physiol. Psychol, 44, 557-564.

Ganz H., Kappen F., 1980. Contribution to the control of the locomotor activity of the dog. Method, Find, Exp, Clin, Pharmacol, 2, 7-15.

Hite M., Hanson H.M., Bohidar N.R., Conti P.A., Mattis P.A., 1977. Effect of cage size on patterns of activity and health of beagle dogs. Lab. Anim. Sci., 27, 60-64.

Hunt J. McV., Schlosserg H., 1939. General activity in the male white rat. J. comp. Psychol., 28, 23-38.

KAYSER C., 1978. Analyse du rythme circadien de l'activité motrice du rat blanc par l'emploi de plusieurs agents pharmacologiques agissant sur les monoamines cérébrales. Arch. int. Physiol. Biochim., 86, 1099-1116.

Le Magnen J., Tallon S., 1966. La périodicité spontanée de la prise d'aliments ad libitum du rat blanc. J. Physiol. (Paris), 58, 323-349.

LEVITSKY D.A., 1974 Feeding conditions and intermeal relationships. Physiol. Behav., 12, 779-787.

Mathews S.R., Finger F.W., 1966. Direct observation of the rat's activity during food deprivation. Physiol. Behav., 1, 85-88.

Mony L., Charpentier J., Dauza T.M., Chanelet J., 1978. Méthode d'analyse et de mesure des comportements. Application à l'activité globale nocturne du rat. C.R. Soc. Biol., 172, 437-441.

Natelson B.H., Bonbright J.C., 1978. Patterns of eating and drinking in monkeys when food and water are free and when they are earned. Physiol. Behav., 21, 201-213.

O'Connor W.J., PotTs D.J., 1969. The external water exchanges of normal laboratory dogs. Quart. J. exp. Physiol., 54, 244-265.

Richter C.P., 1927. Animal behavior and internal drives. Quart. Rev. Biol., 2, 307-343.

SANDerson J.D., VanderweEle D.A., 1975. Analysis of feeding patterns in normal and vagotomized rabbits. Physiol. Behav., 15, 357-364. 